\title{
AN ERROR ANALYSIS OF STIRLING'S METHOD IN BANACH SPACES
}

\author{
IOANNIS K. ARGYROS
}

\begin{abstract}
The method of nondiscrete mathematical induction is applied to Stirling's method. The method yields a very simple proof of the convergence and error estimates which are generally better than those given in the literature.
\end{abstract}

\section{Introduction}

Consider the equation

$$
x=F(x)
$$

where $F$ is a nonlinear operator on a Banach space $E$. Stirling's method, namely

$$
x_{n+1}=x_{n}-\left[I-F^{\prime}\left(F\left(x_{n}\right)\right)\right]^{-1}\left[x_{n}-F\left(x_{n}\right)\right], \quad n=0,1,2, \cdots
$$

for some $x_{0} \in E$, has been used to approximate a fixed point $x^{*}$ of equation (1) in [7].

L. Rall has given sufficient conditions for the convergence of (2) in [7], under the condition that the Fréchet-derivative of $F$ is such that

$$
\left\|F^{\prime}(x)\right\| \leq \alpha<1
$$

Received April 11, 1990; revised November 10, 1992.

(1980) A.M.S. classification codes: 65H10, 65.

Key words and phrases: Stirling's method, nondiscrete mathematical induction, Banach space. 
Stirling's method can be viewed as a combination of the method of successive substitutions and Newton's method as it can easily be seen for a real function. In terms of computational effort, Stirling's and Newton's methods required essentially the same labor per step, as each requires the evaluation of $F$, $F^{\prime}$ and the inversion of a linear operator, assuming that $F^{\prime}$ and its derivative are evaluated independently.

In the first part of this paper we prove convergence, under the assumption that the above condition holds in a ball centered at the initial iterate $x_{0}$ and of a certain radius $R>0$.

In the second part of this paper using the method of nondiscrete mathematical induction we study two important problems [4], [5]:

(a) We give sufficient conditions, in terms of $F$ and the initial iterate $x_{0}$, for the convergence of the sequence generated by (2) to a fixed point of the equation (1).

(b) We find a priori and a posteriori estimates for the distances $\left\|x_{n}-x^{*}\right\|$.

We show that our approach gives estimates on the distances $\left\|x_{n}-x^{*}\right\|$ which are in general better than those given by L. Rall [7] and others.

A numerical example is also provided. To make the paper self-contained we reproduce here part of the results of [4].

\section{Nondiscrete induction and Stirling's method}

Let $T$ be either the positive real axis or an interval of the form $T=\{r \in \mathbb{R}$; $0<r<b\}$.

Definition. A function $w: T \rightarrow T$ is called a rate of convergence on $T$ if the series

$$
\sigma(r)=\sum_{n=0}^{\infty} w^{(n)}(r)
$$

is convergent for each $r \in T$, where the iterates $w^{(n)}$ of $w$ are defined as follows:

$$
w^{(0)}(r)=r, w^{(n+1)}(r)=w\left(w^{(n)}(r)\right), \quad n=0,1,2, \cdots
$$


The functions $w$ and $\sigma$ from the above definition satisfy obviously the following functional equation:

$$
\sigma(w(r))=\sigma(r)-r, \quad r \in T .
$$

Now let $E$ be a Banach space. If $x \in E$ and $r$ is a positive real number, we shall denote by $\bar{U}(x, r)$ the closed ball with center $x$ and radius $r$. Let $F$ be an operator on $E, x_{0} \in E$ and consider the iterative procedure given by (2).

Suppose we can attach to the pair $\left(F, x_{0}\right)$ a rate of convergence $w$ on an interval $T$ and a family of sets $Z(r) \subset E, r \in T$, such that the followig conditions are satisfied

$$
x_{0} \in Z\left(r_{0}\right) \text { for a certain } r_{0} \in T \text {, }
$$

$$
(r \in T \text { and } x \in Z(r)) \Rightarrow x-\left[I-F^{\prime}(F(x))\right]^{-1}(x-F(x)) \in \bar{U}(x, r) \cap Z(w(r)) \text {. }
$$

From (6), and from repeated application of (7), it follows that the sequence generated (2) satisfies the relations

$$
\begin{gathered}
x_{n} \in Z\left(w^{(n)}\left(r_{0}\right)\right) \\
\left\|x_{n}-x_{n-1}\right\| \leq w^{(n)}\left(r_{0}\right) .
\end{gathered}
$$

The last inequality shows that the distances between the consecutive terms of the sequence given by (2) are majorized by the terms of a convergent series. Hence, the sequence $\left\{x_{n}\right\}, n=1,2, \ldots$ is convergent. Let us denote by $x^{*}$ its limit. From (9) we obtain the following estimates for the distances between $x_{n}$ and $x^{*}$ :

$$
\left\|x_{n}-x^{*}\right\| \leq \sigma\left(w^{(n)}\left(r_{0}\right)\right) .
$$

The above estimates may be regarded as a priori estimates. In order to obtain a posteriori estimates, let us suppose that the following relation is satisfied:

$$
x_{n-1} \in Z\left(\left\|x_{n}-x_{n-1}\right\|\right) .
$$

Then, taking $x_{n-1}$ instead of $x_{0}$ and $\left\|x_{n}-x_{n-1}\right\|$ instead of $r_{0}$, we infer as above that

$$
\left\|x_{n}-x^{*}\right\| \leq d\left(\left\|x_{n}-x_{n-1}\right\|\right)
$$


where we have denoted

$$
d(r)=\sigma(r)-r
$$

Summing up what we have stated above we obtain the following theorem:

Theorem 1. (a) If we can attach to the pair $\left(F, x_{0}\right)$ a rate of convergence $w$ on an interval $T$ and a family oj sets $Z(r) \subset E, r \in T$ such that conditions (6) and (7) are satisfied, then the iteration generated by (2) yields a sequence $\left\{x_{n}\right\}, n=1,2, \cdots$ which converges to a fixed point $x^{*}$ of equation (1), such that the relations (8), (9), and (10) are satisfied.

(b) If, in addition, for a certain $n \in\{1,2, \cdots\}$ the condition (11) is satisfied then for this $n$ inequality (12) is also satisfied.

The above theorem is regarded a corollary of the induction theorem [5].

From now on we assume that the Fréchet-derivative $F^{\prime}$ of $F$ is $K$-Lipschitz continuous in a sufficiently large ball containing the ball $\bar{U}\left(x_{0}, R\right)$.

We now provide sufficient conditions for the existence of a locally unique fixed point $x^{*}$ of equation (1).

Theorem 2. Let $F$ be as in the introduction and $x_{0} \in E$ be such that

(a) For some $R>0$ and all $x, y \in \bar{U}\left(x_{0}, R\right)$

$$
\|F(x)-F(y)\| \leq \alpha\|x-y\|, \quad 0<\alpha<1
$$

(b) The follwing estimates are true:

$$
\begin{gathered}
\left\|F^{\prime}\left(F\left(x_{0}\right)\right)\right\| \leq \gamma \\
m=\min \left(R_{1}, R_{2}, R_{4}\right)>M=\max \left(R_{3}, R_{5}\right)
\end{gathered}
$$


where

$$
\begin{aligned}
& R_{1} \equiv \frac{1-\gamma}{\alpha K}, \\
& \left.R_{2} \equiv \frac{1}{\alpha \beta K}, \quad \beta \geq \| I-F^{\prime}\left(F\left(x_{0}\right)\right)\right)^{-1} \| \\
& R_{3} \equiv \frac{\left\|F\left(x_{0}\right)-x_{0}\right\|}{1-\alpha} \\
& R_{4} \equiv \frac{1-\beta(\alpha+\gamma)}{2 \alpha \beta K}, \\
& R_{5} \equiv \frac{1-\beta(\alpha+\gamma)-\sqrt{1-\beta(\alpha+\gamma)]^{2}-8 \alpha \beta^{2} K\left\|F\left(x_{0}\right)-x_{0}\right\|}}{4 \alpha \beta K}
\end{aligned}
$$

with

$$
\beta(\alpha+\gamma)<1
$$

and

$$
[1-\beta(\alpha+\gamma)]^{2}>8 \alpha \beta^{2} K\left\|F\left(x_{0}\right)-x_{0}\right\|
$$

Then,

(i) if (14) holds for some $R \geq R_{3}$ then equation (1) has a unique fixed point $x^{*} \in \vec{U}\left(x_{0}, R\right)$;

(ii) moreover for $M \leq R<m$ the iterates generated by (2) are well defined, remain in $\bar{U}\left(x_{0}, R\right)$ and converge to $x^{*}$ with

$$
\left\|x_{n+1}-x^{*}\right\| \leq b\left\|x_{n}-x^{*}\right\|
$$

where

$$
b \equiv \frac{\beta(\alpha+\alpha K M+\gamma)}{1-\alpha \beta K M}
$$

Proof. The result in (i) will follow from the contraction mapping principle [see, ex. [6], pp. 65-66] and the two claims:

Claim 1. The operator $F$ is a contraction on $\bar{U}\left(x_{0}, R\right), R_{1} \geq R_{3}$.

Let $x, y \in \bar{U}\left(x_{0}, R\right), R \geq R_{3}$, then

$$
\|F(x)-F(y)\| \leq \alpha\|x-y\|, \quad 0<\alpha<1
$$


by (14) and the claim is justified.

Claim 2. F maps $\bar{U}\left(x_{0}, R\right)$ into itself.

Let $x \in \bar{U}\left(x_{0}, R\right), R \geq R_{3}$, then

$$
\begin{aligned}
\left\|F(x)-x_{0}\right\| & =\left\|F(x)-F\left(x_{0}\right)+F\left(x_{0}\right)-x_{0}\right\| \\
& \leq\left\|F(x)-F\left(x_{0}\right)\right\|+\left\|F\left(x_{0}\right)-x_{0}\right\| \\
& \leq \alpha R+\left\|F\left(x_{0}\right)-x_{0}\right\| .
\end{aligned}
$$

That is, $\left\|F(x)-x_{0}\right\| \leq R$ if

$$
\alpha R+\left\|F\left(x_{0}\right)-x_{0}\right\| \leq R
$$

which is true by the choice of $R$ and that completes the proof of Claim 2 .

To show (ii) we first need to compute certain quantities.

For $x \in \bar{U}\left(x_{0}, R\right), R<R_{1}$ we have

$$
\begin{aligned}
\left\|F^{\prime}(F(x))\right\| & =\left\|F^{\prime}(F(x))-F^{\prime}\left(F\left(x_{0}\right)\right)+F^{\prime}\left(F\left(x_{0}\right)\right)\right\| \\
& \leq K\left\|F(x)-F\left(x_{0}\right)\right\|+\left\|F^{\prime}\left(F\left(x_{0}\right)\right)\right\| \\
& \leq \alpha K R+\left\|F^{\prime}\left(F\left(x_{0}\right)\right)\right\|<1
\end{aligned}
$$

by the choice of $R$.

By (15) the linear operator $\left(I-F^{\prime}\left(F\left(x_{0}\right)\right)\right)$ is invertible. Using the identity $I-F^{\prime}(F(x))=\left(I-F^{\prime}\left(F\left(x_{0}\right)\right)\right)\left[I+\left(I-F^{\prime}\left(F\left(x_{0}\right)\right)\right)^{-1}\left(F^{\prime}\left(F\left(x_{0}\right)\right)-F^{\prime}(F(x))\right)\right]$ for $x \in \bar{U}\left(x_{0}, R\right), R<R_{2}$ and the Banach Lemma on invertible operators we derive that the linear operator $\left(I-F^{\prime}(F(x))\right)$ is invertible on $\bar{U}\left(x_{0}, R\right), R<R_{2}$ and

$$
\|\left(I-F^{\prime}(F(x))^{-1} \| \leq \frac{\left\|\left(I-F^{\prime}\left(F\left(x_{0}\right)\right)\right)^{-1}\right\|}{1-\alpha K R\left\|\left(I-F^{\prime}\left(F\left(x_{0}\right)\right)\right)^{-1}\right\|}=\frac{\beta}{1-\alpha \beta K R} .\right.
$$

Let us now assume that $x_{K} \in \bar{U}\left(x_{0}, R\right)$ for $K=0,1,2, \cdots, n$ and some $R$, $M \leq R<m$. We show that $x_{n+1} \in \bar{U}\left(x_{0}, R\right)\left(x_{n+1}\right.$ is well defined by the choice of $R$ ). 
We have by (2) that

$$
\begin{aligned}
& x_{n+1}-x_{0} \\
= & \left(x_{n}-x_{0}\right)-\left[I-F^{\prime}\left(F\left(x_{n}\right)\right)\right]^{-1}\left(x_{n}-F\left(x_{n}\right)\right) \\
= & {\left[I-F^{\prime}\left(F\left(x_{n}\right)\right)^{-1}\left[\left(F\left(x_{n}\right)-F\left(x_{0}\right)\right)-F^{\prime}\left(F\left(x_{n}\right)\right)\left(x_{n}-x_{0}\right)+\left(F\left(x_{0}\right)-x_{0}\right)\right] .\right.}
\end{aligned}
$$

By taking norms in both sides of (22) we show that

$$
\left\|x_{n+1}-x_{0}\right\| \leq R
$$

if

$$
\left\|x_{n+1}-x_{0}\right\| \leq \frac{\beta}{1-\alpha \beta K R}\left[\alpha R+(K \alpha R+\gamma) R+\left\|F\left(x_{0}\right)-x_{0}\right\|\right] \leq R
$$

which is true by the choice of $R$.

Finally, to show (19) we take norms in both sides of the identity

$$
x_{n+1}-x^{*}=\left[I-F^{\prime}\left(F\left(x_{n}\right)\right)\right]^{-1}\left[\left(F\left(x_{n}\right)-F\left(x^{*}\right)\right)-F^{\prime}\left(F\left(x_{n}\right)\right)\left(x_{n}-x^{*}\right)\right]
$$

to obtain, using (20) and (21) that

$$
\begin{aligned}
\left\|x_{n+1}-x^{*}\right\| & \leq \frac{\beta}{1-\alpha \beta K R}\left[\alpha\left\|x_{n}-x^{*}\right\|+(\alpha K R+\gamma)\left\|x_{n}-x^{*}\right\|\right] \\
& \leq \frac{\beta}{1-\alpha \beta K R}(\alpha+\alpha K R+\gamma)\left\|x_{n}-x^{*}\right\| \\
& \leq b\left\|x_{n}-x^{*}\right\| .
\end{aligned}
$$

Note that by the choice of $M, 0<b<1$, which shows that the sequence generated (2) remains in $\bar{U}\left(x_{0}, R\right)$ and converges to $x^{*}$.

That completes the proof of the theorem.

The above theorem shows that the convergence of the sequence given by (2) to $x^{*}$ is only linear. In the next theorem we show that we can do even better.

Theorem 3. Let $F$ be as in the introduction and assume that:

(a) the conditions (14), (15), (17) and (18) of Theorem 2 hold; 
(b) the following estimates are true

$$
\bar{m}=\min \left(m, R_{6}\right)>M
$$

where

$$
R_{6}=\frac{2}{\beta K(1+4 \alpha)}
$$

and $x_{0} \in E$ is such that

$$
h_{s}=\frac{\beta K(1+2 \alpha)}{2(1-\alpha \beta K R)} M<1 .
$$

Then the sequence generated by (2) is well defined, remains in $\bar{U}\left(x_{0}, R\right)$ for $M \leq R<\bar{m}$ and converges quadratically to a unique fixed point $x^{*}$ of equation (1) in $\bar{U}\left(x_{0}, R\right)$ with

$$
\left\|x_{n}-x^{*}\right\| \leq\left(h_{s}\right)^{2^{n}-1} M, \quad n=0,1,2, \cdots .
$$

Proof. The existence of the solution $x^{*}$ and the sequence generated by (2) follows immediately as in Theorem 2. Let $x_{k} \in \bar{U}\left(x_{0}, R\right)$, for $M \leq R<\bar{m}$, $k=0,1,2, \cdots, n$, then

$$
\left\|x^{*}-F\left(x_{n}\right)\right\|=\left\|F\left(x^{*}\right)-F\left(x_{n}\right)\right\| \leq \alpha\left\|x_{n}-x^{*}\right\|,
$$

and

$$
\left\|x_{n}-F\left(x_{n}\right)\right\|=\left\|\left(x_{n}-x^{*}\right)+\left(F\left(x^{*}\right)-F\left(x_{n}\right)\right)\right\| \leq(1+\alpha)\left\|x_{n}-x^{*}\right\| .
$$

As

$$
\begin{aligned}
& F\left(x_{n}\right)-F\left(x^{*}\right)-F^{\prime}\left(F\left(x_{n}\right)\right)\left(x_{n}-x^{*}\right) \\
= & \int_{0}^{1}\left[F^{\prime}\left(t x_{n}+(1-t) x^{*}\right)-F^{\prime}\left(t F\left(x_{n}\right)+(1-t) F\left(x_{n}\right)\right]\left(x_{n}-x^{*}\right) d t\right.
\end{aligned}
$$

it follows that

$$
\begin{aligned}
& \left\|F\left(x_{n}\right)-F\left(x^{*}\right)-F^{\prime}\left(F\left(x_{n}\right)\right)\left(x_{n}-x^{*}\right)\right\| \\
\leq & K \int_{0}^{1}\left[t\left\|x_{n}-F\left(x_{n}\right)\right\|+(1-t)\left\|x^{*}-F\left(x_{n}\right)\right\|\right]\left\|x_{n}-x^{*}\right\| d t \\
= & \frac{1}{2} K\left[\left\|x_{n}-F\left(x_{n}\right)\right\|+\left\|F\left(x_{n}\right)-x^{*}\right\|\right]\left\|x_{n}-x^{*}\right\| .
\end{aligned}
$$


Therefore, with the above majorizations, the identity

$$
x_{n+1}-x^{*}=\left[I-F^{\prime}\left(F\left(x_{n}\right)\right)\right]^{-1}\left[\left(F\left(x_{n}\right)-F\left(x^{*}\right)\right)-F^{\prime}\left(F\left(x_{n}\right)\right)\left(x_{n}-x^{*}\right)\right]
$$

becomes

$$
\begin{aligned}
\left\|x_{n+1}-x^{*}\right\| & \leq \frac{\beta K(1+2 \alpha)}{2(1-\alpha \beta K R)}\left\|x_{n}-x^{*}\right\|^{2} \\
& \leq \frac{\beta K(1+2 \alpha)}{2(1-\alpha \beta K M)}\left\|x_{n}-x^{*}\right\|^{2} .
\end{aligned}
$$

Inequality (27) follows by mathematical induction from (30) and the satisfaction of (26) guarantees that the sequence generated by (2) remains in $\bar{U}\left(x_{0}, R\right)$ and converges to $x^{*}$.

The proof of the theorem is now completed.

We now state the Theorems 1 and 4 of [7] for comparison with Theorems 2 and 3 respectively.

Theorem 4. If

$$
\left\|F^{\prime}(x)\right\| \leq \alpha \leq \frac{1}{3}, \text { for all } x \in \bar{U}\left(x_{0},\left[\frac{1+2 a}{1-a}\right]\left\|x_{0}-F\left(x_{0}\right)\right\|\right),
$$

then the sequence generated by (2) converges to the unique fixed point $x^{*}$ of (1) for any $x_{0} \in E$. Furthermore,

$$
\left\|x^{*}-x_{n}\right\| \leq\left[\frac{2 \alpha}{1-\alpha}\right]^{n} \frac{\left\|x_{0}-F\left(x_{0}\right)\right\|}{1-\alpha}, \quad n=0,1,2, \cdots
$$

and

$$
\left\|x_{n+1}-x^{*}\right\| \leq \frac{2 \alpha}{1-\alpha}\left\|x_{n}-x^{*}\right\|
$$

Theroem 5. If $F^{\prime}$ is Lipschitz continuous with constant $K$, and $\left\|F^{\prime}(x)\right\| \leq$ $\alpha<1$, for all $x \in \bar{U}\left(x_{0}, \frac{2\left\|x_{0}-F\left(x_{0}\right)\right\|}{1-a}\right)$, then the sequence generated by (2) converges to $x^{*}$ starting from any $x_{0} \in E$ such that

$$
\bar{h}_{s}=\frac{K}{2} \cdot \frac{1+2 \alpha}{1-\alpha} \frac{\left\|x_{0}-F\left(x_{0}\right)\right\|}{1-\alpha}<1
$$


furthermore, the convergence is quadratic, with

$$
\left\|x_{n}-x^{*}\right\| \leq \frac{K}{2} \frac{1+2 \alpha}{1-\alpha}\left\|x_{n-1}-x^{*}\right\|^{2}, \quad n=1,2, \cdots
$$

and

$$
\left\|x_{n}-x^{*}\right\| \leq\left(\bar{h}_{s}\right)^{2^{n}-1} \frac{\left\|x_{0}-F\left(x_{0}\right)\right\|}{1-\alpha}, \quad n=0,1,2, \cdots
$$

Remark 1 . Note that since $\mathcal{B}, \gamma$ can be chosen such that

$$
\mathcal{B} \leq \frac{1}{1-a} \text { and } \gamma \leq a
$$

the condition in theorem 4 implies (17) but not conversely.

We can now prove a consequence of Theorem 1 for iteration (2) and equation (1).

Theorem 6. Let $F$ be as in the introdiction and $x_{0} \in E$ be such that:

(a) the following inequalities are satisfied:

$$
\begin{gathered}
\left\|\left(I-F^{\prime}\left(F\left(x_{0}\right)\right)\right)^{-1}\right\| \leq \beta \\
0 \leq \delta \leq 1-\left\|\left(I-F^{\prime}\left(F\left(x_{0}\right)\right)\right)^{-1}\right\| \cdot\left(\alpha+\left\|F^{\prime}\left(F\left(x_{0}\right)\right)\right\|\right) \\
0<\bar{\gamma} \leq 1-\left\|F^{\prime}\left(F\left(x_{0}\right)\right)\right\| ; \\
b\left\|F\left(x_{0}\right)-x_{0}\right\| \leq r_{0} ;
\end{gathered}
$$

and

$$
8 \alpha \beta r_{0} K<\delta^{2} .
$$

(b) There exists a rate of convergence $w$ with corresponding continuous monotonic function $\sigma$ and positive continuous monotonic functions $A, B, C$ such that

$$
\begin{gathered}
C(w(r)) \leq C(r)-\alpha \operatorname{Kr} A(r) \\
\frac{A(r)}{1-\alpha \operatorname{Kr} A^{2}(r)} \leq A(w(r)) \\
1-\alpha \operatorname{Kr} A^{2}(r)>0
\end{gathered}
$$




$$
\begin{gathered}
\frac{B(r)-2 \alpha K r A^{2}(r)}{1-\alpha K r A^{2}(r)} \geq B(w(r)) \\
w(r) \geq \frac{1}{2} \Pi(2+A(r)) A(r) A(w(r)) r^{2} \\
A\left(r_{0}\right)=\beta \\
B\left(r_{0}\right)=\delta \\
C\left(r_{0}\right)=\bar{\gamma}
\end{gathered}
$$

and

$$
\sigma(0)=0
$$

Then the iteration generated by (2) is well defined on $\bar{U}\left(x_{0}, R\right), M \leq \mathbb{R} \leq \bar{m}$ and it converges to a unique fixed point $x^{*}$ of equation $(1)$ in $\bar{U}\left(x_{0}, R\right)$.

Moreover, the following estimates are true:

$$
\begin{aligned}
\left\|x_{n}-x_{n-1}\right\| & \leq A\left(x^{(n-1)}\left(r_{0}\right)\right) w^{(n-1)}\left(r_{0}\right), \quad n=1,2, \cdots \\
\left\|x_{n-1}-x_{0}\right\| & \leq \sigma\left(r_{0}\right)-\sigma\left(\left\|x_{n}-x_{n-1}\right\|\right), \quad n=2,3, \cdots \\
\left\|x_{0}-x^{*}\right\| & \leq \sigma\left(r_{0}\right) \\
\left\|x_{n}-x^{*}\right\| & \leq \sum_{k=1}^{n-1} A\left(w^{(k)}\left(r_{0}\right)\right) w^{(K)}\left(r_{0}\right)+\sigma\left(r_{0}\right), \quad n=1,2, \cdots
\end{aligned}
$$

and

$$
\left\|x_{n}-x^{*}\right\| \leq d\left(\left\|x_{n}-x_{n-1}\right\|\right)
$$

where $d$ is as defined in (13).

Proof. The proof of this theorem is based on Theorem 1.

We attach to the iteration given by (2) the rate of convergence $w$ with corresponding function $\sigma$, and the family of sets $Z(r)=\left\{x \in E ;\left\|x-x_{0}\right\| \leq\right.$ $\sigma\left(r_{0}\right)-\sigma(r),\left(I-F^{\prime}(F(x))\right)$ is boundedly invertible, $\left\|\left(I-F^{\prime}(F(x))\right)^{-1}\right\| \leq A(r)$, $0 \leq B(r) \leq 1-\|\left(I-F^{\prime}(F(x))^{-1} \| \cdot\left(\alpha+\left\|F^{\prime}(F(x))\right\|\right)\right.$,

$$
\left.0<C(r) \leq 1-\left\|F^{\prime}(F(x))\right\|,\left\|\left(I-F^{\prime}(F(x))\right)^{-1}(F(x)-x)\right\| \leq r\right\}, \quad r \in T
$$


where $A, B$ and $C$ satisfy the hypotheses in (b) above.

According to Theorem 1, we have to show that the condition (6), (7) and (11) are satisfied.

The hypotheses of the theorem imply that $Z\left(r_{0}\right)=\left\{x_{0}\right\}$ so that (6) is satisfied. Now let $x$ be an element of $Z(r)$ and denote

$$
y=x-\left[I-F^{\prime}(F(x))\right]^{-1}[x-F(x)] .
$$

We will show that $y \in Z(w(r))$. Using (5) and (49), we have

$$
\left\|y-x_{0}\right\| \leq\|y-x\|+\left\|x-x_{0}\right\| \leq r+\sigma\left(r_{0}\right)-\sigma(r)=\sigma\left(r_{0}\right)-\sigma(w(r)) .
$$

We now have,

$$
\begin{aligned}
\left\|F^{\prime}(F(y))\right\| & =\left\|\left(F^{\prime}(F(y))-F^{\prime}(F(x))\right)+F^{\prime}(F(x))\right\| \\
& \leq K\|F(y)-F(x)\|+\left\|F^{\prime}(F(x))\right\| \\
& \leq \alpha \operatorname{Kr} A(r)+\left\|F^{\prime}(F(x))\right\|
\end{aligned}
$$

that is,

$$
\begin{aligned}
1-\left\|F^{\prime}(F(y))\right\| & \geq 1-\left\|F^{\prime}(F(x))\right\|-\alpha K r A(r) \\
& \geq C(r)-\alpha K r A(r) \geq C(w(r))
\end{aligned}
$$

by (38).

Also

$$
I-F^{\prime}(F(y))=\left(I-F^{\prime}(F(x))\right)\left[I+\left(I-F^{\prime}(F(x))\right)^{-1}\left(F^{\prime}(F(x))-F^{\prime}(F(y))\right)\right],
$$

that is

$$
\begin{aligned}
\left\|\left(I-F^{\prime}(F(y))\right)^{-1}\right\| & \geq \frac{\left.\| I-F^{\prime}(F(x))\right)^{-1} \|}{1-\left\|\left(I-F^{\prime}(F(x))\right)^{-1}\right\|^{2} \alpha K r} \\
& \leq \frac{A(r)}{1-A(r) \alpha \operatorname{Kr} A(r)} \leq A(w(r))
\end{aligned}
$$

by (34) and (40).

Moreover, we can easily obtain

$$
\begin{aligned}
& 1-\left(\alpha+\left\|F^{\prime}(F(y))\right\|\right)\left\|\left(I-F^{\prime}(F(y))\right)^{-1}\right\| \\
\geq & 1-\left[\alpha K r A(r)+\left(\alpha+\left\|F^{\prime}(F(x))\right\|\right)\right] \frac{\left\|\left(I-F^{\prime}(F(x))\right)^{-1}\right\|}{1-\left\|\left(I-F^{\prime}(F(x))\right)^{-1}\right\| \alpha K r A(r)} \\
\geq & \frac{\beta(r)-2 \alpha K r A^{2}(r)}{1-\alpha K r A^{2}(r)} \geq B(w(r))
\end{aligned}
$$


by (41).

Furthermore, since by (50), we can write

$$
\begin{aligned}
y-F(y) & =y-F(y)+\left(I-F^{\prime}(F(x))\right)(x-y)-(x-F(x)) \\
& =F(x)-F(y)-F^{\prime}(F(x))(x-y) .
\end{aligned}
$$

As in (29) we can easily obtain

$$
\begin{aligned}
& \|\left(I-F^{\prime}(F(y))^{-1}(y-F(y)) \|\right. \\
\leq & \frac{1}{2} A(w(r)) K[\|x-F(x)\|+\|y-F(x)\|]\|x-y\| \\
\leq & \frac{1}{2} K[2 r+r A(r)] r A(r) \\
\leq & \frac{1}{2} K(2+A(r)) A(r) A(w(r)) r^{2} \leq w(r)
\end{aligned}
$$

by (42).

Thus we have proved that the condition (7) is also satisfied. Hence, the first part of Theorem 1 assures that the sequence generated by (2) converges to a point $x^{*}$ and that the relations (8), (9) and (10) are satisfied.

From the fact that $x_{n-1} \in Z\left(w^{(n-1)}\left(r_{0}\right)\right)$ and from the monotonicity of the functions $\sigma, A, B, C$ we obtain

$$
\begin{aligned}
\left\|x_{n}-x_{n-1}\right\| & \leq\left\|\left[I-F^{\prime}\left(F\left(x_{n-1}\right)\right)\right]^{-1}\right\|\left\|x_{n-1}-F\left(x_{n-1}\right)\right\| \\
& \leq A\left(w^{(n-1)}\left(r_{0}\right)\right) w^{(n-1)}\left(r_{0}\right) \\
\left\|x_{n-1}-x_{0}\right\| & \leq \sigma\left(r_{0}\right)-\sigma\left(w^{(n-1)}\left(r_{0}\right)\right) \\
& \leq \sigma\left(r_{0}\right)-\sigma\left(\left\|x_{n}-x_{n-1}\right\|\right) .
\end{aligned}
$$

Thus, the relation (11) is also verified for $n=1,2, \cdots$.

We conclude the proof of the theorem noting that the fact $x^{*}=F\left(x^{*}\right)$ may be obtained by taking in (50) $y=x_{n+1}, x=x_{n}$ and using the continuity of $F$.

Note, that in practice, the functions $w, \sigma, A, B$ and $C$ cannot easily be found. However, if they are foound then the error bounds obtained in the previous theorem are expected to be sharper than the ones obtained in Theorems $2-5$. 
One way (but not the only one) to make this apparent is to strengthen the assumptions of Theorem 6.

This is actually done in the following theorem.

Theorem 7. Assume:

(a) the operator $F^{\prime}$ is Lipschitz continuous with constant $K$ and

$$
\|F(x)-F(y)\| \leq \alpha\|x-y\|, \quad 0<\alpha<1 \text { for all } x, y \in E
$$

(b) There exists $x_{0} \in E$ and $r_{0} \geq 0$ such that

$$
\frac{\left\|x_{0}-F\left(x_{0}\right)\right\|}{1-\alpha} \leq r_{0}<\min \left[\frac{2(1-\alpha)}{k(1+2 \alpha)}, \frac{1}{q}\right]
$$

where

$$
q=\frac{k(3-2 \alpha)}{2(1-\alpha)}
$$

Then the iteration generated by (2) is well defined on $E$ and it converges to a fixed point $x^{*}$ of equation (1).

Moreover, the following estimates are true:

$$
\begin{aligned}
\left\|x_{n}-x_{n-1}\right\| & \leq w^{(n-1)}\left(r_{0}\right) \\
\left\|x_{n-1}-x_{0}\right\| & \leq \sigma\left(r_{0}\right)-\sigma\left(w^{(n-1)}\left(r_{0}\right)\right) \leq \sigma\left(r_{0}\right)-\sigma\left(\left\|x_{n}-x_{n-1}\right\|\right) \\
\left\|x_{n}-x^{*}\right\| & \leq \sigma\left(w^{(n)}\left(r_{0}\right)\right), \quad n=1,2, \cdots
\end{aligned}
$$

and

$$
\left\|x_{n}-x^{*}\right\| \leq d\left(\left\|x_{n}-x_{n-1}\right\|\right)
$$

where $d$ is as defined in (13) with

$$
w^{(n)}(r)=\frac{1}{q}(q r)^{2^{n}}, \quad n=0,1,2, \cdots
$$

and

$$
\sigma(r)=\frac{1}{q} \sum_{m=0}^{\infty}(q r)^{2^{m}}
$$




\section{Proof. The proof will follow using theorem 5.}

We attach to the iteration (2) the rate of convergence $w$ given by (52) and the family of sets

$$
Z(r)=\left\{x \in E ;\left\|x-x_{0}\right\| \leq \sigma\left(r_{0}\right)-\sigma(r),\|x-F(x)\| \leq(1-\alpha) r\right\}
$$

where $\sigma$ is given by (53).

The hypotheses of the theorem imply that $Z\left(r_{0}\right)=\left\{x_{0}\right\}$ so that (6) is satisfied. Now let $x$ be an element of $Z(r)$ and denote $y$ as in (50).

We want to show that $y \in Z(w(r))$. Using (5) and (54) we have

$$
\left\|y-x_{0}\right\| \leq\|y-x\|+\left\|x-x_{0}\right\| \leq r+\sigma\left(r_{0}\right)-\sigma(r)=\sigma\left(r_{0}\right)-\sigma(w(r)) .
$$

Using (50) we also have as in (29)

$$
y-F(y)=F(x)-F(y)-F^{\prime}(F(x))(x-y)
$$

and easily

$$
\left\|\left(I-F^{\prime}(F(y))\right)^{-1}(y-F(y))\right\| \leq \frac{K}{2(1-\alpha)}(\|x-F(x)\|+\|y-F(x)\|)\|x-y\|
$$

since

$$
\begin{aligned}
& \|\left(I-F^{\prime}(F(y))^{-1} \| \leq \frac{1}{1-\alpha}\right. \\
\leq & \frac{K}{2(1-\alpha)}(r+(1-\alpha) r+(1-\alpha) r) r \\
\leq & q r^{2}=w(r) .
\end{aligned}
$$

Thus, we have proved that the condition (7) is also satisfied. Hence, the first part of Theorem 1 assures the fact that the sequence generated by (2) converges to a point $x^{*}$ and that the relations (8), (9) and (10) are satisfied. By continuity, iteration (2) given $x^{*}=F\left(x^{*}\right)$.

From the fact that $x_{n-1} \in Z\left(w^{(n-1)}\left(r_{0}\right)\right)$ and from the monotonicity of $\sigma$ we get

$$
\left\|x_{n}-x_{n-1}\right\|=\left\|\left(I-F^{\prime}\left(F\left(x_{n-1}\right)\right)\right)^{-1}\left(x_{n-1}-F\left(x_{n-1}\right)\right)\right\| \leq w^{(n-1)}\left(r_{0}\right)
$$


and

$$
\left\|x_{n-1}-x_{0}\right\| \leq \sigma\left(r_{0}\right)-\sigma\left(w^{(n-1)}\left(r_{0}\right)\right) \leq \sigma\left(r_{0}\right)-\sigma\left(\left\|x_{n}-x_{n-1}\right\|\right) .
$$

Thus, the relation (11) is also verified for $n=1,2, \cdots$.

The rest of the theorem follows as Theorem 6 and that completes the proof of this theoem.

Remark 2. (a) It can easily be seen that our error estimates given by (51) are eventually sharper than the ones given by Rall in (32) if

$$
g r_{0}=\frac{K(3-2 \alpha)}{2(1-\alpha)^{2}}\left\|x_{0}-F\left(x_{0}\right)\right\|<\frac{K(1+2 \alpha)}{2(1-\alpha)^{2}}\left\|x_{0}-F\left(x_{0}\right)\right\|
$$

that is if

$$
\frac{1}{2}<\alpha<1
$$

(b) It is well known that there are cases when Stirling's method compares favorably with Newton's method. Moreover there are examples when Stirling's method applies when Newton's fails (see ex. [7] and the references there).

For some applications it may be convenient to have the function $\sigma$ expressed not as a series of the form (53). In that case we suggest the following alternative:

Lemma. Let $v, w$ be real numbers such that $v>0, w \geq 0$ and $T=\{r \in$ $\left.\mathbb{R} / 0<r<\frac{1}{v}\right\}$. Then the function

$$
w(r)=\left[\left(x_{0}-r\right)^{2}-w^{2}\right]\left[2\left(x_{0}-r\right)+v\left(\left(x_{0}-r\right)^{2}-w^{2}\right)\right]^{-1}
$$

with

$$
x_{0}=x_{0}(r)=\left[r+\left(r^{2}+(1-v r)^{2} w^{2}\right)^{\frac{1}{2}}\right](1-v r)^{-1}
$$

is a rate of convergence on $T$ and the corresponding function $\sigma$ is given by

$$
\sigma(r)=x_{0}-w
$$

Proof. Let $w$ consider the real polynomial defined on a closed finite interval centered at $x_{0}$, on which (14) is satisfied and

$$
F(x)=\frac{1}{2} v\left(x^{2}-w^{2}\right)+x
$$


and let $x_{0}$ be as defined above. The Stirling's iteration (2) becomes

$$
x_{n+1}=x_{n}-\frac{x_{n}^{2}-w^{2}}{2 x_{n}+v\left(x_{n}^{2}-w^{2}\right)}
$$

yields a decreasing sequence $\left\{x_{n}\right\}, n=0,1,2, \cdots$ which converges the fixed point $w$ of $F$.

We now have that

$$
x_{0}-x_{1}=r
$$

and by taking

$$
w(r)=x_{1}-x_{2}
$$

we obtain the expression $w$ defined above.

Finally it can easily be seen that

$$
w^{(n)}(r)=x_{n}-x_{n+1}, \quad n=0,1,2, \cdots
$$

and consequently $\sigma(r)=x_{0}-w$. That completes the proof.

Following the ideas in [4] for Newton's method and using the functions $w$ and $\sigma$ defined in the Lemma, one can produce a theorem similar to theorem 6 above.

However we leave that to the motivated reader and we complete this paper with some applications.

\section{Applications}

In what follows we shall compare the estimates (27) and (51) with (32) - on a numerical example.

Let $F$ be the real polynomial given by the formula

$$
F(x)=\frac{1}{8}\left(x^{2}-2\right)
$$

with (14) holding only on the interval $[-2.2,1.8]$, say. 
Then $K=.25$ and $\alpha=.55$. Taking $x_{0}=-.2$ we obtain

$$
\begin{array}{ll}
\multicolumn{1}{c}{\left\|x_{0}-F\left(x_{0}\right)\right\|=.045,} \\
\gamma=.06125, & b=.94228504 \\
R_{1}=6.827272727, & R_{2}=7.718181828, \\
R_{3}=.1, & R_{4}=1.63636364, \\
R_{5}=.593285138, & 1-b(\alpha+\gamma)=.42402826, \\
m=R_{4}, & M=R_{5}, \\
R_{6}=2.653125003, & \bar{m}=m,
\end{array}
$$

and $h_{s}$ given by (26) is such that

$$
.15896865 \leq h_{s} \leq .186233 .
$$

With the above values it can easily be seen that the conditions of theorems 3 and 7 are satisfied. The conditions of theorem 5 apply here also.

Moreover, iteration (2) becomes

$$
x_{n+1}=\frac{4 x_{n}^{2}-x_{n}^{3}+2 x_{n}-8}{34-x_{n}^{2}}, \quad n=0,1,2, \cdots .
$$

Note that the unique fixed point of $(55)$ in $[-2,2,1.8]$ is $x^{*}=-.242640687$ and using (56) with $x_{0}=-.2$ we can now tabulate the following results:

\begin{tabular}{|c|l|l|l|l|}
\hline$n$ & \multicolumn{1}{|c|}{$x_{n}$} & $\begin{array}{c}\text { Results by Rall } \\
(32)\end{array}$ & $\begin{array}{c}\text { Results by Argyros } \\
(51)\end{array}$ & $\begin{array}{c}\text { Results by Argryos } \\
(27)\end{array}$ \\
\hline 0 & $x_{0}=-.2$ & .1 & .105292479 & .593285138 \\
1 & $x_{1}=-.2424028$ & .00583333 & .0052924791093 & .094313737 \\
2 & $x_{2}=-.24264068$ & $1.984953704 \cdot 10^{-5}$ & $1.470133149 \cdot 10^{-5}$ & .002383405447 \\
\hline
\end{tabular}

The above table clearly indicates the superiority of the estimates (51) when compared with (32) in this case.

Finally another example can be given. Consider the real function $F$ given by

$$
F(x)= \begin{cases}\frac{1}{8} x, & x \leq 1 \\ \frac{1}{32}\left(x^{2}+2 x+1\right), & 1 \leq x \leq 3 \\ \frac{1}{4}(x-1), & x \geq 3\end{cases}
$$


Then it can easily be seen that $\alpha=\frac{1}{4}, K=\frac{1}{16}$ and with $x_{0}=.1,\left|x_{0}-F\left(x_{0}\right)\right|=$ .0875. The hypotheses of theorem 7 are now satisfied with the above values and (2) gives

$$
x_{1}=0=x^{*}
$$

which is the unique fixed point of equation (57) in this case.

Remark 3. By Remark 2 (a) it can easily be seen that by choosing $\alpha$ much closer to 1 than to $\frac{1}{2}$ our comparison results demonstrated in the table will improve even furtherover the corresponding ones given by $\mathbb{R}$ all.

\section{References}

[1] I. K. Argyros, "On Newton's method and nondiscrete mathematical induction." Bulll. Austral. Math. Soc., Vol. 38 (1988), 131-140.

[2] L. V. Kantorovich, "Functional analysis and applied mathematics." Uspekhi Mat. Nauk. 3, (1948), 89-185.

[3] J. M. Ortega and W. C. Rheinboldt, "Iterative solution of nonlinear equations in several variables." New York, Academic Press, 1970.

[4] F. A. Potra and V. Ptak, "Sharp error bounds for Newton's process." Numer. Math. 34, (1980), 63-72.

[5] __ "Nondiscrete induction and iterative processes." Pitman Publ. Boston, 1984.

[6] L. B. Rall, "Computational solution of nonlinear operator equations." John Wiley Publ. 1969.

[7] "Convergence of Stirling's method in Banach spaces." Aeq. Math., Vol. 12, (1975), 12-19.

[8] J. Stirling, "Methods differentials: sive tractatus de summatione et interpolatione serierum infinitarum." W. Boyer, London, 1730.

[9] R. A. Tapia, "The Kantorovich theorem for Newton's method", Amer. Math. Soc. Monthly 78, (1971), 389-392.

Cameron University, Department of Mathematics, Lawton, OK 73505-6377, U. S. A. 